\title{
Metformide Hydrochloride/Pioglitazone Hydrochloride Extended-Release Tablet
}

National Cancer Institute

\section{Source}

National Cancer Institute. Metformide Hydrochloride/Pioglitazone Hydrochloride

Extended-Release Tablet. NCI Thesaurus. Code C129316.

An extended-release (ER) tablet composed of the hydrochloride salt form of the biguanide metformin and the hydrochloride salt form of the thiazolidinedione piog litazone, with antihyperglycemic activity. Upon oral administration and although the exact mechanism of action has yet to be fully elucidated, metformin inhibits complex I (NADPH:ubiquinone oxidoreductase) of the mitochondrial respiratory chain and increases the cellular AMP to ATP ratio leading to activation of AMP-activated protein kinase (AMPK). This modulates AMPK-mediated transcription of target genes, which prevents hepatic gluconeogenesis, decreases intestinal absorption of glucose, enhances insulin sensitivity and fatty acid oxidation, and increases glucose uptake and utilization in target tissues. Piog litazone binds to and activates the transcription factor peroxisome proliferator-activated receptor gamma (PPAR-gamma), thereby increasing the transcription of insulin-responsive genes. This enhances insulin sensitivity of insulindependent tissues, decreases insulin resistance, enhances peripheral glucose utilization, and decreases hepatic glucose output. Altogether, this lowers blood glucose levels. 